\title{
Transmissibility of cholera: In vivo-formed biofilms and their relationship to infectivity and persistence in the environment
}

\author{
Shah M. Faruque*t, Kuntal Biswas*, S. M. Nashir Udden*, Qazi Shafi Ahmad*, David A. Sack*, G. Balakrish Nair*t, \\ and John J. Mekalanos ${ }^{\text {t* }}$
}

*Molecular Genetics Laboratory, International Centre for Diarrhoeal Disease Research, Bangladesh, Dhaka-1212, Bangladesh; and ₹Department of Microbiology and Molecular Genetics, Harvard Medical School, 200 Longwood Avenue, Boston, MA 02115

Contributed by G. Balakrish Nair, February 15, 2006

The factors that enhance the waterborne spread of bacterial epidemics and sustain the epidemic strain in nature are unclear. Although the epidemic diarrheal disease cholera is known to be transmitted by water contaminated with pathogenic Vibrio cholerae, routine isolation of pathogenic strains from aquatic environments is challenging. Here, we show that conditionally viable environmental cells (CVEC) of pathogenic $V$. cholerae that resist cultivation by conventional techniques exist in surface water as aggregates (biofilms) of partially dormant cells. Such CVEC can be recovered as fully virulent bacteria by inoculating the water into rabbit intestines. Furthermore, when $V$. cholerae shed in stools of cholera patients are inoculated in environmental water samples in the laboratory, the cells exhibit characteristics similar to CVEC, suggesting that CVEC are the infectious form of $V$. cholerae in water and that CVEC in nature may have been derived from human cholera stools. We also observed that stools from cholera patients contain a heterogenous mixture of biofilm-like aggregates and free-swimming planktonic cells of $V$. cholerae. Estimation of the relative infectivity of these different forms of $V$. cholerae cells suggested that the enhanced infectivity of $V$. cholerae shed in human stools is largely due to the presence of clumps of cells that disperse in vivo, providing a high dose of the pathogen. The results of this study support a model of cholera transmission in which in vivo-formed biofilms contribute to enhanced infectivity and environmental persistence of pathogenic $V$. cholerae.

cholera epidemic | Vibrio cholerae | conditionally viable environmental cells | waterborne disease

To oxigenic Vibrio cholerae of the O1 or O139 serogroup are the causative agents of cholera and belong to a group of organisms whose major habitat is aquatic ecosystems (1-3). Epidemics of cholera occur frequently in many developing countries of Asia, Africa, and Latin America $(4,5)$, and these occurrences coincide with increased prevalence of the causative $V$. cholerae strain in the aquatic environment (6). However, the concentration of toxigenic $V$. cholerae usually detected in surface water by standard culture techniques is far less than that required to induce infection and cause clinical disease under experimental conditions in volunteers challenged with $V$. cholerae $(7,8)$. This discrepancy between the required infectious dose and the apparent concentration of $V$. cholerae in surface water fostering an epidemic has not been adequately explained.

Various hypotheses have been proposed to explain the mode of persistence of pathogenic $V$. cholerae in the aquatic environment and to suggest that the true prevalence of the pathogen in natural water may be considerably higher than that observed by standard culture methods. For example, it has been proposed that $V$. cholerae can exist in the aquatic environment in a viable but nonculturable state, which by definition is a condition in which cells are incapable of forming a colony on commonly used media and thus cannot be recovered from the water by standard culture techniques $(1,2)$. This hypothesis stems from observa- tions that water carries vibrio-shaped cells that appear to be antigenically related to $V$. cholerae $\mathrm{O} 1$ strains, as determined by a fluorescent anti-O1 antibody-based procedure (9), but the cells are apparently not culturable. However, without a verified resuscitation procedure, it has not been possible to ascertain whether the same strain producing viable but nonculturable cell counts in the water is responsible for cholera epidemics. Both $V$. cholerae $\mathrm{O} 1$ and the non-O1/non-O139 strains that do not carry the major virulence genes also exist in the environment, further complicating this challenge (10).

Another explanation for the low recovery of $V$. cholerae from environmental water by conventional culture techniques might relate to the cellular organization of the bacteria in the water. Environmental bacteria are known to form biofilms, which are surface-associated communities of bacterial cells, to enhance their survival under adverse conditions (11). $V$. cholerae also has been postulated to form biofilms in association with animate and inanimate surfaces $(12,13)$. Because the transition between a planktonic form of the bacteria and biofilms is a complex and highly regulated process, conventional culture techniques may not permit accurate estimation of $V$. cholerae that exist as biofilms in the environment. The role of such biofilms in the epidemiology of cholera is also unclear, but presumably these cells can reconvert to active bacteria and cause subsequent outbreaks of the disease.

We recently showed that a significant proportion of toxigenic $V$. cholerae $\mathrm{O} 1$ cells in the aquatic environment exist in a "conditionally viable" state and require appropriate growth conditions for their detection, and we designated these cells "conditionally viable environmental cells" (CVEC) (14). However, the public health importance of this survival form of $V$. cholerae $\mathrm{O} 1$ depends on whether these cells are infectious and naturally reconvertible to active bacteria. Here, we demonstrate that $V$. cholerae CVEC are aggregates of partially dormant cells that resuscitate to normal, viable bacteria, both under appropriate in vitro conditions and in the intestinal environment of adult rabbits. We further show that the CVEC are derived from biofilm-like multicellular clumps of $V$. cholerae shed in human stools, and that such biofilms are responsible for enhanced infectivity of $V$. cholerae. This study thus provides insight into factors that promote the rapid spread of cholera epidemics, as well as into the persistence in the environment of $V$. cholerae with epidemic potential.

\section{Results}

Characterization of $\boldsymbol{V}$. cholerae CVEC. Our method for demonstrating the presence of CVEC of $V$. cholerae in water samples that

Conflict of interest statement: No conflicts declared.

Abbreviations: CVEC, conditionally viable environmental cells; AST, antibiotic selection technique; $\mathrm{Sm}^{\mathrm{R}}$, streptomycin resistance; $\mathrm{Nal}{ }^{\mathrm{R}}$, nalidixic acid resistance; cfu, colony-forming units; BP, bile/peptone; TTGA, taurocholate-tellurite-gelatin agar.

†To whom correspondence may be addressed. E-mail: faruque@icddrb.org, gbnair@ icddrb.org, or shannon_humphreys@hms.harvard.edu.

( ) 2006 by The National Academy of Sciences of the USA 
Table 1. Differential response of environmental $\boldsymbol{V}$. cholerae 01 cells to streptomycin and nalidixic acid in enrichment culture, despite carrying genetic determinants for resistance to both antibiotics

\begin{tabular}{|c|c|c|c|c|}
\hline \multirow[b]{2}{*}{$\begin{array}{l}\text { Date of } \\
\text { sample } \\
\text { collection }\end{array}$} & \multirow[b]{2}{*}{$\begin{array}{l}\text { Sample } \\
\text { ID }\end{array}$} & \multicolumn{3}{|c|}{$\begin{array}{c}V . \text { cholerae } 01 \text { counts }(\mathrm{cfu} / \mathrm{ml}) \text { on TTGA plates } \\
\text { containing streptomycin, after enrichment for } 6 \mathrm{~h} \\
\text { under various conditions* }\end{array}$} \\
\hline & & $\begin{array}{l}\text { BP medium } \\
\text { without } \\
\text { antibiotic }\end{array}$ & $\begin{array}{l}\text { BP medium } \\
\text { containing } \\
\text { streptomycin }^{+}\end{array}$ & $\begin{array}{l}\text { BP medium } \\
\text { containing } \\
\text { nalidixic acid }{ }^{\ddagger}\end{array}$ \\
\hline Sept. 6, 2005 & 1714 & $3.3 \times 10^{5}$ & 0 & $1.8 \times 10^{4}$ \\
\hline Sept. 11, 2005 & 1724 & $8.6 \times 10^{4}$ & 0 & $8.4 \times 10^{3}$ \\
\hline Oct. 2, 2005 & 1753 & $1.2 \times 10^{4}$ & 0 & $2.6 \times 10^{4}$ \\
\hline Oct. 2, 2005 & 1754 & $1.2 \times 10^{4}$ & 0 & $1.8 \times 10^{4}$ \\
\hline Oct. 9, 2005 & 1764 & $2.0 \times 10^{4}$ & 0 & $1.2 \times 10^{4}$ \\
\hline Nov. 28, 2005 & 1824 & $1.6 \times 10^{4}$ & $2 \times 10^{1}$ & $1.5 \times 10^{4}$ \\
\hline Nov. 28, 2005 & 1825 & $8.0 \times 10^{2}$ & 0 & $1.1 \times 10^{3}$ \\
\hline Dec. 6, 2005 & 1834 & $2.4 \times 10^{3}$ & 0 & $1.7 \times 10^{3}$ \\
\hline Dec. 13, 2005 & 1845 & $2.0 \times 10^{2}$ & 0 & $2.7 \times 10^{2}$ \\
\hline Dec. 13, 2005 & 1847 & $6.8 \times 10^{3}$ & 0 & $9.2 \times 10^{3}$ \\
\hline
\end{tabular}

cfu, colony-forming units; TTGA, taurocholate-tellurite-gelatin agar.

*Each value represents the mean of at least three independent observations with different aliquots of the same sample.

${ }^{\dagger}$ A colony count of zero means that no colonies were detected on plating $100-\mu l$ aliquots of enriched culture. In theory, the concentration of bacteria should be $<10 \mathrm{cfu} / \mathrm{ml}$.

${ }^{\ddagger}$ All $V$. cholerae strains selected on nalidixic acid plates were later found to be resistant to streptomycin and to carry the SXT element.

only rarely yielded viable $V$. cholerae by conventional culture exploited the antibiotic-resistant property of the bacteria and was termed the "antibiotic selection technique" (AST). The strain of $V$. cholerae known to have caused recent cholera epidemics in Bangladesh carries the SXT element $(15,16)$, which encodes resistance to multiple antibiotics, including streptomycin, sulfamethoxazole, and trimethoprim. This strain is also constitutively resistant to nalidixic acid. We used a selective medium containing streptomycin $(70 \mu \mathrm{g} / \mathrm{ml})$ to isolate $V$. cholerae $\mathrm{O} 1$ with epidemic potential from surface water samples that appeared negative for the organisms in conventional assays. However, we observed that a prerequisite to the success of the AST procedure was that the water sample be cultured for at least $5 \mathrm{~h}$ in bile/peptone (BP; $1 \%$ peptone $/ 0.5 \%$ taurocholic acid $/ 1 \%$ $\mathrm{NaCl}, \mathrm{pH}$ 9.0) medium (enrichment), in the absence of streptomycin, before aliquots were plated on the antibiotic-containing plates. When streptomycin was added directly to the enrichment medium, very few $(0.03-0.8 \%) \mathrm{V}$. cholerae $\mathrm{O} 1$ colonies could be recovered (see Table 3, which is published as supporting information on the PNAS web site). This finding led us to explore whether $V$. cholerae CVEC were impaired in expressing streptomycin resistance $\left(\mathrm{Sm}^{\mathrm{R}}\right)$ prior to being revitalized by initial culturing in the absence of streptomycin.

In the epidemic strain of $V$. cholerae, $\mathrm{Sm}^{\mathrm{R}}$ is mediated through enzymatic modification of the antibiotic by enzymes encoded by the $\operatorname{str} A B$ genes of the SXT element $(15,16)$. Cells that are defective in protein synthesis are unlikely to produce the required enzymes, despite carrying their genetic determinants, and hence are unable to express $\mathrm{Sm}^{\mathrm{R}}$ phenotype. Furthermore, because streptomycin itself is an inhibitor of protein synthesis, a temporary or partial impairment of $\mathrm{Sm}^{\mathrm{R}}$ is likely to be further augmented by the presence of streptomycin, resulting in complete inhibition of protein synthesis and eventual cell death. Our results indicated that, in the CVEC state, $V$. cholerae cells possibly undergo at least a partial impairment in protein synthesis and hence cannot express $\mathrm{Sm}^{\mathrm{R}}$ unless they are first revitalized by growing in nutrient medium, in the absence of the antibiotic. We verified this assumption by exploiting the nalidixic acid resistance $\left(\mathrm{Nal}^{\mathrm{R}}\right)$ of the same bacteria, because $\mathrm{Nal}^{\mathrm{R}}$ is not directly dependent on protein synthesis, but is associated with mutations in the gyrA gene encoding the GyrA subunit of DNA gyrase (17). We conducted assays in which nalidixic acid was used in the enrichment media instead of streptomycin, before selection of the organism on antibiotic-containing plates. As expected, use of nalidixic acid during the enrichment did not drastically reduce the recovery of cells (Table 1), suggesting that, in contrast to $\mathrm{Sm}^{\mathrm{R}}$, the $\mathrm{Nal}^{\mathrm{R}}$ phenotype remained largely unchanged in the CVEC form of $V$. cholerae. These results further supported our assumption that the CVEC form of $V$. cholerae found in water represents a metabolically impeded state of the bacteria, as evidenced by their lack of expression of $\mathrm{Sm}^{\mathrm{R}}$.

V. cholerae CVEC Exist as Multicellular Clumps. We further examined the cellular organization and metabolic status of CVEC by conducting a series of experiments that included in vitro growth kinetics assay and fluctuation analysis. In the growth kinetics assay, samples were removed from the enrichment mixture in BP medium at regular intervals and plated on antibiotic-containing selective medium for the target $V$. cholerae strain. We sought to understand whether the rate of increase in colony counts with time is comparable with that predicted on the basis of an increase in cell number by binary fission, or whether the increase is considerably higher, suggesting possible activation of dormant $V$. cholerae cells. The results showed a minimal increase in viable cell number during the first $4 \mathrm{~h}$ of enrichment; subsequently, however, viable cells increased drastically faster than would be consistent with normal bacterial growth kinetics (Table 2). This initial lack of viable cell counts was also inconsistent with the duration of the lag phase of normal bacterial growth, as was observed with laboratory-grown $V$. cholerae in control experiments. Thus, the growth kinetics study suggested that $V$. cholerae CVEC are living cells, but they require at least $4 \mathrm{~h}$ of enrichment to assume normal growth and colony formation. The resuscitation kinetics are also consistent with results reported by Baker et al. (18) on the properties of nutrient-starved $V$. cholerae cells, which required incubation for $\approx 5 \mathrm{~h}$ in rich medium to reassume 
Table 2. Growth kinetics assays for $V$. cholerae 01 CVEC in environmental waters

\begin{tabular}{|c|c|c|c|c|c|c|c|}
\hline \multirow{2}{*}{$\begin{array}{l}\text { Date of } \\
\text { sample } \\
\text { collection }\end{array}$} & \multirow{2}{*}{$\begin{array}{l}\text { Sample } \\
\text { ID }\end{array}$} & \multicolumn{6}{|c|}{$\begin{array}{l}\text { V. cholerae } 01 \text { counts }(\mathrm{cfu} / \mathrm{ml})^{* \dagger} \text { on TTGA plates containing } \\
\text { streptomycin, after enrichment for } 1-6 \mathrm{~h} \text { in BP medium }\end{array}$} \\
\hline & & $1 \mathrm{~h}$ & $2 \mathrm{~h}$ & $3 \mathrm{~h}$ & $4 \mathrm{~h}$ & $5 \mathrm{~h}$ & $6 \mathrm{~h}$ \\
\hline Aug. 2, 2005 & 1664 & 0 & 0 & 0 & $8.9 \times 10^{2}$ & $2.2 \times 10^{4}$ & $7.5 \times 10^{4}$ \\
\hline Aug. 9, 2005 & 1674 & 0 & 0 & 0 & $1.2 \times 10^{3}$ & $4.1 \times 10^{4}$ & $1.2 \times 10^{5}$ \\
\hline Aug. 14, 2005 & 1684 & 0 & 0 & 0 & $2.4 \times 10^{3}$ & $7.6 \times 10^{4}$ & $4.0 \times 10^{5}$ \\
\hline Oct. 9, 2005 & 1753 & 0 & 0 & $2.0 \times 10^{1}$ & $2.5 \times 10^{2}$ & $2.5 \times 10^{3}$ & $5.4 \times 10^{3}$ \\
\hline Oct. 9, 2005 & 1754 & 0 & 0 & 0 & $5.0 \times 10^{1}$ & $6.0 \times 10^{2}$ & $3.3 \times 10^{3}$ \\
\hline Oct. 17, 2005 & 1774 & 0 & 0 & $6.0 \times 10^{1}$ & $1.2 \times 10^{3}$ & $1.1 \times 10^{4}$ & $3.1 \times 10^{4}$ \\
\hline Nov. 28, 2005 & 1824 & 0 & 0 & $2.1 \times 10^{1}$ & $3.9 \times 10^{2}$ & $7.4 \times 10^{3}$ & $2.7 \times 10^{4}$ \\
\hline Nov. 28, 2005 & 1825 & 0 & 0 & 0 & $2.4 \times 10^{1}$ & $3.3 \times 10^{2}$ & $1.2 \times 10^{3}$ \\
\hline Dec. 6, 2005 & 1834 & 0 & 0 & 0 & $1.1 \times 10^{2}$ & $2.6 \times 10^{3}$ & $1.5 \times 10^{4}$ \\
\hline Dec. 13, 2005 & 1845 & 0 & 0 & 0 & $1.6 \times 10^{2}$ & $1.9 \times 10^{3}$ & $7.3 \times 10^{3}$ \\
\hline Dec. 13, 2005 & 1847 & 0 & 0 & $2.0 \times 10^{1}$ & $2.4 \times 10^{2}$ & $3.6 \times 10^{3}$ & $1.2 \times 10^{4}$ \\
\hline \multicolumn{2}{|c|}{$\begin{array}{l}\text { Laboratory-grown } \\
\text { environmental strain } \\
\text { diluted in PBS }\end{array}$} & $8.0 \times 10^{1}$ & $3.6 \times 10^{2}$ & $1.7 \times 10^{3}$ & $7.4 \times 10^{3}$ & $2.9 \times 10^{4}$ & $1.5 \times 10^{5}$ \\
\hline \multicolumn{2}{|c|}{$\begin{array}{l}\text { Laboratory-grown } \\
\text { clinical strain diluted } \\
\text { in PBS }\end{array}$} & $2.5 \times 10^{1}$ & $9.0 \times 10^{1}$ & $4.2 \times 10^{2}$ & $2.2 \times 10^{3}$ & $9.2 \times 10^{3}$ & $4.5 \times 10^{4}$ \\
\hline
\end{tabular}

*A colony count of zero means that no colonies were detected on plating of $100-\mu$ l aliquots of enriched culture. In theory, the concentration should be $<10$ colonies per $\mathrm{ml}$.

tThe rate of increase in viable cells during enrichment of $V$. cholerae in environmental water samples was considerably faster than would be consistent with normal bacterial growth kinetics between 3 and 5 h, suggesting that cells might have generated by activation of dormant $V$. cholerae cells.

normal size and shape under laboratory conditions. Thus, the CVEC present in water samples characterized in the current study appear to be viable $V$. cholerae cells that are perhaps similar to the nutrient-starved cells described by Baker et al.

Dispersion of clumped viable cells present in biofilms might also explain the sudden increase in viable counts that occurred after 4-5 $\mathrm{h}$ of enrichment cultivation of CVEC. Thus, we further investigated whether CVEC are planktonic cells or are present in clumps that disperse into planktonic cells during the AST procedure. Fluctuation analysis (19) was used to determine whether there was an asymmetric spatial distribution of CVEC in the water samples. In this assay, water samples were diluted in BP medium, and the diluted samples were broken down further into multiple small aliquots. Each aliquot was assayed for the appearance of viable cells during the AST procedure by using the growth kinetics assay described above. Although most of the aliquots were found to be negative for $V$. cholerae, the assays revealed "jackpots" of $V$. cholerae cells in small number of aliquots, and detectable cells increased dramatically in the tubes containing these aliquots (Table 4, which is published as supporting information on the PNAS web site). Therefore, it appears that the CVEC exist as clumps of potentially viable cells that disperse into viable planktonic cells during the enrichment process. Taken together, these results suggest that CVEC are aggregates of metabolically impeded cells, and that the resuscitation process involves both dispersion of clumps and activation of cells.

To observe the presence of clumped cells, we conducted fluorescent anti-O1 antibody-based microscopic examination of aliquots of water samples, and this assay showed the presence of clumps of cells that reacted with the antibody (Fig. 1). Interestingly, some of these cells showed a coccoid morphology, in agreement with the morphology of starved $V$. cholerae cells reported by Baker et al. (18). To further document that the clumped cells were alive, we conducted assays involving enrichment on agar plates instead of in broth medium. We plated aliquots of the same water on a nylon membrane placed on BP agar plates; after $5 \mathrm{~h}$ of enrichment, the membrane was transferred to the selective plate [taurocholate-tellurite-gelatin agar
(TTGA) containing streptomycin]. Large "macrocolonies" formed on the plates (Fig. 4, which is published as supporting information on the PNAS web site) and were confirmed to be composed of $V$. cholerae $\mathrm{O} 1$ cells by biochemical tests and agglutination with $V$. cholerae O1-specific antiserum. Ribotyping and antibiotic resistance profiles confirmed that these cells were identical to the strain responsible for recent epidemics of cholera. We conclude that the unusual morphology of these colonies reflects the attachment of large number of vibrios to macroscopic material present in the aquatic samples.

CVEC Convert to Infectious Bacteria in the Rabbit Intestine. In view of previous studies suggesting that the epidemiological cycle of
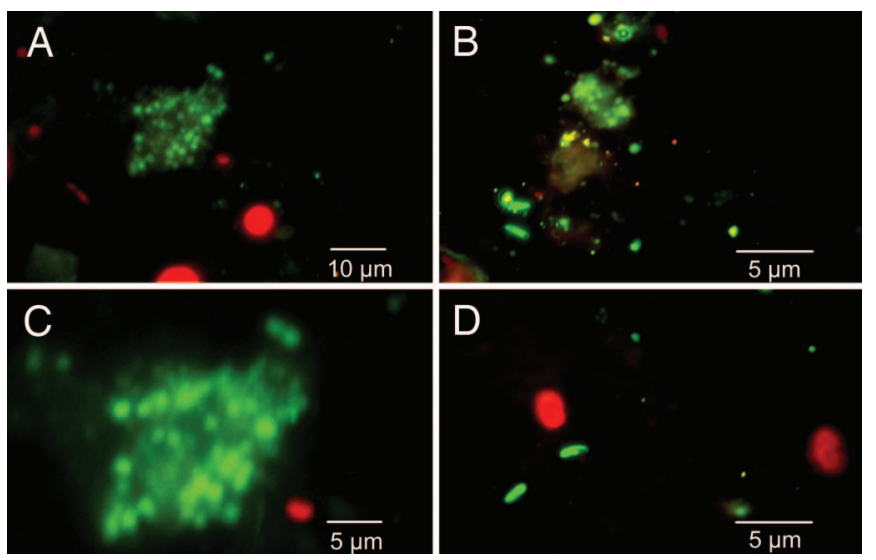

Fig. 1. Fluorescent antibody-based detection of $V$. cholerae 01 CVEC in water samples. Shown are aggregates of CVEC reacting with $V$. cholerae O1-specific monoclonal antibody $(A-C)$, as well as planktonic cells of $V$. cholerae $\mathrm{O} 1(B$ and $D)$. C shows high magnification of a CVEC clump that appears to contain both coccoid (starved) and rod-shaped $V$. cholerae 01 cells. The corresponding water samples produced viable V. cholerae 01 in the AST assay, as well as in the ileal loop assay in rabbits, and further analyses confirmed that these isolates were identical to the strain responsible for recent epidemics of cholera in Bangladesh. 

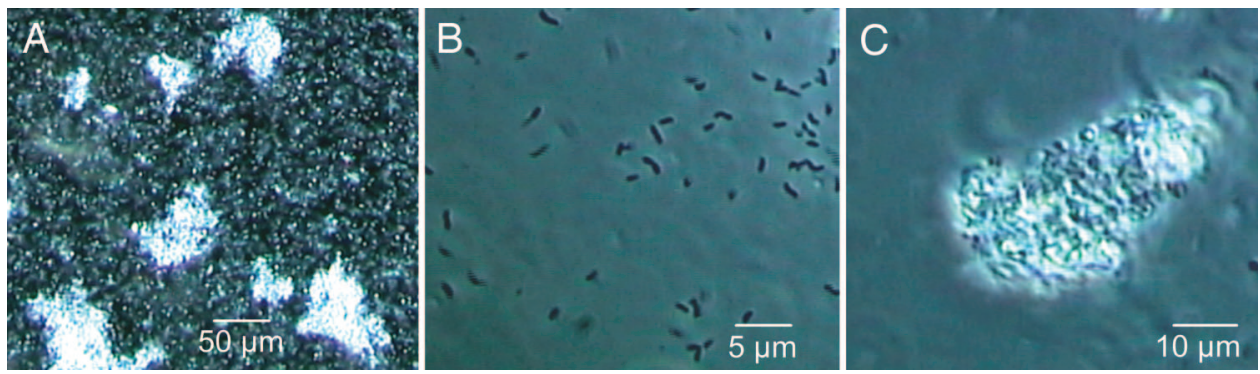

Fig. 2. Micrographs of different fractions of human cholera stools. Both planktonic cells and biofilm-like clumps of $V$. cholerae cells are present in unfractionated stool $(A)$. The planktonic cells $(B)$ were separated from the clumps $(C)$ by differential centrifugation, and the precipitated clumps were further washed to remove free cells. Numerous $V$. cholerae cells in multiple layers were found tightly attached to debris in stools, forming biofilms (C).

pathogenic $V$. cholerae is supported by both environmental and host factors (4), we examined whether the CVEC form of $V$. cholerae would convert to viable pathogenic bacteria in the mammalian intestinal environment. Adult rabbits have long been used as a suitable model to study the pathogenesis of $V$. cholerae (20). We inoculated rabbit ileal loops with aliquots of water sample that were negative for $V$. cholerae $\mathrm{O} 1$ in conventional culture but appeared to contain CVEC in the AST assay. After $18 \mathrm{~h}$ of incubation, $46.7 \%$ of the ileal loops were found to contain between $1.2 \times 10^{2}$ and $5.7 \times 10^{6}$ cells of viable toxigenic $V$. cholerae $\mathrm{O} 1$ (Table 5, which is published as supporting information on the PNAS web site), and $38.8 \%$ of the $V$. cholerae-positive ileal loops also showed fluid accumulation, which is characteristic of enterotoxin production by the bacteria. Thus, it appears that CVEC are converted to viable virulent bacteria in the mammalian intestinal environment. These results suggest that CVEC forms of environmental $V$. cholerae are likely to be infectious in humans, and because CVEC exist as multicellular aggregates, human victims are likely to receive a significantly high dose of the pathogen by ingesting CVEC. The observation that only some aliquots of the same water are able to cause a fluid-accumulation response in the rabbit intestine (Table 5) may also reflect the asymmetric spatial distribution of the CVEC clumps in the water.

CVEC-Like Properties of $\boldsymbol{V}$. cholerae Excreted in Human Stools. The rapid spread of cholera epidemics is known to be fostered by surface waters through contamination of water by fecal material from cholera patients. To understand the possible origin of the CVEC found in water, we designed experiments to test whether $V$. cholerae shed in cholera stools show CVEC-like properties when inoculated in otherwise $V$. cholerae O1-negative environmental water samples. We collected stools from cholera patients and initially examined the stools by dark-field microscopy for the presence of characteristic highly motile $V$. cholerae organisms. Aliquots of the stools were inoculated in environmental water samples that had previously tested negative for $V$. cholerae $\mathrm{O} 1$ and O139. The $\mathrm{pH}$ of the water samples was between 7.0 and 7.5. After the samples had been maintained for $6 \mathrm{~h}$ and $72 \mathrm{~h}$ at room temperature, aliquots of each water sample were analyzed with the same AST procedure and growth kinetics assay we used to test environmental water samples. The results suggested that at least a portion of the $V$. cholerae organisms shed in stools had converted to CVEC after inoculation in water (Table 6, which is published as supporting information on the PNAS web site), as evidenced by their loss of streptomycin resistance while retaining the $\mathrm{Nal}^{\mathrm{R}}$ phenotype. Interestingly, growth kinetics of cells in these samples also resembled those of naturally occurring CVEC (Table 7, which is published as supporting information on the PNAS web site). However, this transformation of $V$. cholerae in stools to the CVEC form was not observed when stools left for $6 \mathrm{~h}$ without dilution in water were subjected to the same assay (data not shown). Similarly, when stools were diluted in PBS ( $\mathrm{pH}$ 7.2) instead of water, the conversion to the CVEC form was not observed (Table 7). Because environmental fresh water is mostly hypotonic, the conversion to CVEC may have been driven, at least in part, by hypoosmotic stress. Lack of optimum $\mathrm{NaCl}$ concentration might also have contributed to the process, because many $V$. cholerae metabolic functions are powered by $\mathrm{Na}^{+}$ gradient $(21,22)$.

In Vivo-Formed Biofilms Are Responsible for Enhanced Infectivity of V. cholerae Shed in Human Stools. Because $V$. cholerae in human stools, when inoculated in environmental water samples, showed characteristics identical to CVEC, which are multicellular aggregates, we conducted microscopic examination of 18 stool samples from different cholera patients to determine the cellular organization of $V$. cholerae present in the stools. Although a typical cholera stool is generally described as watery (i.e., "rice-water" stool), all stools we examined also appeared to contain particulate matter. Phase-contrast microscopy confirmed that, in addition to planktonic cells, each of these stools contained aggregates of cells attached to debris, forming biofilm-like structures of various sizes (Fig. 2). The presence of these biofilms generally resulted in an asymmetric distribution of $V$. cholerae cells in the stools.

Merrell et al. (23) reported that $V$. cholerae present in cholera stools appeared to be hyperinfectious for infant mice, compared with a laboratory strain grown in vitro to stationary phase. They used microarray analysis to suggest that the effect might be related to transcriptional changes in the organism. Because our studies indicated that the stools of cholera victims contain vibrios in more than one morphological state, we assumed that the apparent hyperinfectivity of stool vibrios could also be due to factors other than genetic regulation of virulence-associated factors. For example, the presence of clumped cells might result in underestimation of input colony counts during competition assays with the reference laboratory-grown strain to determine infectivity. To begin to investigate this possibility, we estimated the relative infectivity of the different phenotypic forms of $V$. cholerae present in cholera stools by using differential centrifugation to fractionate stools according to the size of suspended particles and cell clumps (Fig. 2). We then conducted a competition assay with the same reference strain used by Merrell et al. (23), to determine the relative infectivity of the planktonic cells and biofilm-like cells. The reference strain was grown to both logarithmic phase and stationary phase. Interestingly, the average infectivity of the biofilm-like clumped cells from different stools was 12- to 145-fold higher than for the corresponding planktonic cells, even when the stool vibrios competed with the reference strain grown to logarithmic phase (Fig. 3). The highest competitive index (infectivity) observed in a particular assay for $V$. cholerae clumps in stools was 1,560, compared with the logarithmicphase reference strain, and this infectivity was 487 -fold higher than that of the corresponding planktonic cells from the same stool. When they competed with the reference strain grown to a station- 


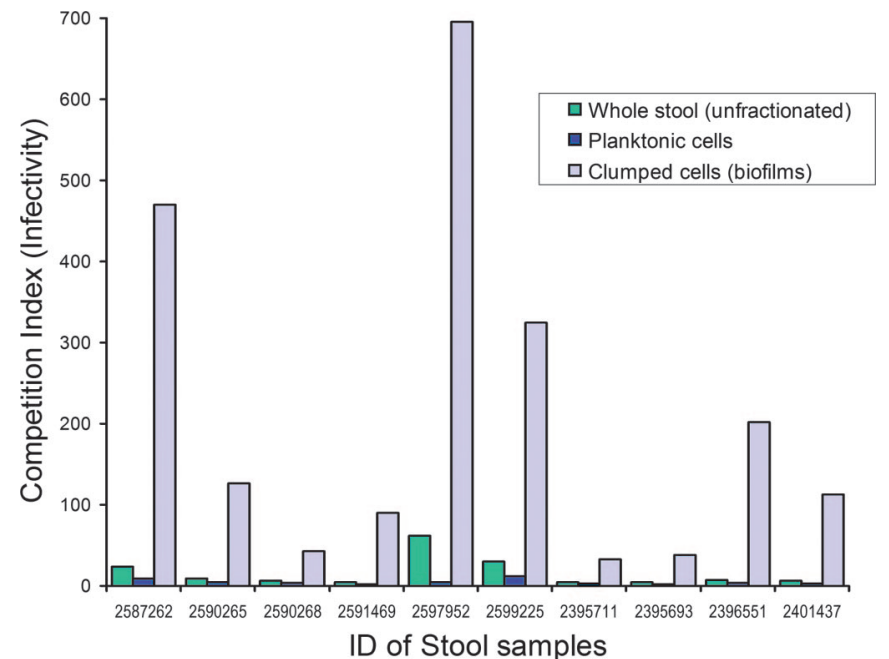

Fig. 3. Infectivity of $V$. cholerae shed in human cholera stools. Shown are mean competition indices of $V$. cholerae 01 present in different fractions of stools from 10 different cholera patients, against a reference strain grown to logarithmic phase. Each mixture of test and reference strains was assayed in 5-10 different mice. Results shown here are for stools that were negative for the presence of any lytic vibriophage.

ary phase, the general infectivity of stool vibrios was 5- to 10 -fold higher than that observed with the reference strain grown to logarithmic phase (Table 8, which is published as supporting information on the PNAS web site). We conclude that the reported hyperinfectivity of $V$. cholerae shed in human stools is largely due to the presence of biofilm-like clumps of cells that disperse in vivo, providing a high dose of the pathogen. However, stools from cholera patients contain complex mixtures of cells, presumably with various mutually exclusive transcriptional programs, that have not been fully characterized.

\section{Conclusions and Implications}

We predict that the results described herein will have profound significance for understanding cholera epidemiology. Our results indicate that the surface waters in a cholera-endemic area carry high concentrations of pathogenic $V$. cholerae as clumps or biofilms of CVEC. Although the $V$. cholerae concentration determined by standard culture may generally appear to be less than the required infectious dose, we predict that human victims are likely to contract cholera by ingesting clumps of CVEC, because these clumps may provide a sufficiently high dose to cause infection. We have shown that environmental water that is apparently negative for virulent $V$. cholerae by conventional enrichment methods, but contains CVEC, can be introduced into rabbit ileal loops and yield virulent $V$. cholerae strains after $18 \mathrm{~h}$ of in vivo incubation. Similarly, humans who ingest clumps of CVEC may resuscitate $V$. cholerae and even amplify the strain. Such an individual could become an index cholera case that begins a cholera epidemic.

Studies by Merrell et al. (23) provided evidence that passage through the human host alters critical phenotypic properties that promote the transmission of pathogens. Our studies confirm that the enhanced infectivity of $V$. cholerae in human stools is largely due to the presence in the stools of $V$. cholerae biofilms formed in vivo. Our studies also suggest that $(i)$ when stools from cholera patients are mixed with environmental water, CVEC are formed and (ii) CVEC are infectious in the rabbit model. Regardless of the mechanisms causing it, the fact that $V$. cholerae cells derived from cholera stools can have enhanced infectivity has significant epidemiological implications. A recent study in Bangladesh reported that filtering environmental water through four layers of old sari cloth material before consumption reduced the incidence of cholera among villagers by $48 \%$ (24). The authors of this study proposed that the reduction in cholera cases occurs because the filtration removes large planktonic material to which $V$. cholerae is likely attached (24). We assume that a similar reduction in the incidence of cholera would be observed if $V$. cholerae in the environment exist as CVEC, which are large clumps of cells possibly derived from human stools, because in theory such clumps can also be eliminated by sari cloth filtration.

$V$. cholerae may have at least three different means for adhering to surfaces, depending on whether the organism is within its human host or in an aquatic environment. The toxin-coregulated pilus is important for colonizing the gut of animals and is an important virulence factor $(4,5)$. A second type-IV pilus, mannose-sensitive hemagglutinin, is not required for gut colonization and pathogenesis, but was shown to be required for biofilm formation on abiotic surfaces (25). $V$. cholerae is also known to colonize the chitin (a polymer of $N$-acetyl-D-glucosamine) surfaces of crustaceans $(2,26)$, and toxin-coregulated pilus has recently been suggested to have a role in forming biofilms on chitin (12). Thus, $V$. cholerae appears to adopt different strategies for biofilm formation, depending on its surroundings. Our studies suggest that cholera patients shed high concentrations of $V$. cholerae as in vivo-formed biofilms, and that the shedding of these clumps of cells, which disperse in the gut of the next human victim, appears to be an efficient way of delivering an infectious dose of the pathogen to the victim. The survival of $V$. cholerae, both in the natural environment and in the acidic stomach environment of the human host, is also likely to be enhanced if cells exist in biofilms (13).

Because cholera is a waterborne disease, the better adapted this bacterium is to survival in the aquatic environment the more likely it is to infect the next victim and even cause subsequent outbreaks of disease. Because our study showed that environmental $V$. cholerae exist largely as CVEC biofilms, we propose that the cells in biofilms may be better protected than the corresponding planktonic cells against the hypoosmotic stress of environmental water. Thus, in vivo biofilm formation appears to be an efficient strategy for both the spread of an epidemic and the persistence of the pathogen in water. In agreement with this assumption, our data also suggest that $V$. cholerae can persist in water, both during and between epidemic periods, as clumps of cells that are conditionally viable and can revive into virulent bacteria in the mammalian intestine. The sensitivity or resistance of CVEC to killing by environmental vibriophages represents an area for future study, given that these lytic viruses modulate the dynamics of cholera epidemics (27). Thus, the prediction of cholera epidemics can likely be facilitated through environmental monitoring of vibriophages, CVEC, and factors that control CVEC resuscitation. This study constitutes the latest refinement in our perception of natural phenomena associated with the transmission of cholera by water.

\section{Methods}

Culture of Environmental Water Samples. Previously established sampling sites (14) along three major rivers, one lake, and marshy lands in and around Dhaka city were used. Water samples were collected from these sites every week between January and December 2005 by using sterile containers and were transported to the laboratory for processing within $2 \mathrm{~h}$ of collection. $V$. cholerae were isolated from the samples by using various enrichment and culture techniques, described below, and by using modifications of the AST described in ref. 14. An aliquot $(5.0 \mathrm{ml})$ of each water sample was added to $2.5 \mathrm{ml}$ of $3 \times$ concentrated BP medium and incubated for $6 \mathrm{~h}$ for enrichment of $V$. cholerae. In another set of BP-enrichment cultures, an antibiotic (streptomycin or nalidixic acid) was added at an appropriate concentration. Dilutions of the enrichment culture were plated on TTGA (28) containing streptomycin $(70 \mu \mathrm{g} / \mathrm{ml})$, as well as on TTGA plates devoid of any antibiotic. Suspected Vibrio colonies were picked and subjected to biochemical and 
serological tests to identify $V$. cholerae O1 or O139 (29). The isolates were also saved for additional genetic analyses, including virulence gene content and ribotype.

Growth Kinetics and Fluctuation Tests. In the growth kinetics assay, $100-\mu l$ aliquots of samples were removed from the enrichment mixture of a typical AST procedure at regular intervals and plated on antibiotic-containing selective medium for the target $V$. cholerae strain, to determine the concentration of viable $V$. cholerae cells. In the fluctuation assay, water samples were initially diluted 10-fold in BP medium, and the diluted samples were broken down further into small aliquots. Each aliquot was assayed for the appearance of viable cells during the AST procedure by using the growth kinetics assay. A laboratorygrown $V$. cholerae $\mathrm{O} 1$ strain, diluted in PBS, was used as a positive control. The control strain was similarly inoculated in a reaction mixture, in parallel with each set of assay.

Assays in Rabbits. Multiple aliquots $(1 \mathrm{ml})$ of each water sample were inoculated directly into different ileal loops of adult New Zealand White rabbits as described (11). After $18 \mathrm{~h}$, the rabbits were killed and the loops were examined for fluid accumulation. Ileal loop fluids were collected, and dilutions of the fluids in 10 $\mathrm{mM}$ PBS ( $\mathrm{pH} 7.5$ ) were plated on TTGA plates containing streptomycin. Rabbit ileal loops showing no fluid accumulation were also washed internally with PBS, and the washings were cultured. Suspected Vibrio colonies were picked and tested by biochemical and serological methods (29).

Assays in Cholera Stools. Freshly collected cholera stools from patients who did not receive antibiotics before reporting to the hospital were examined by dark-field microscopy to ascertain the presence of typical highly motile vibrio-shaped organisms. The stools were then diluted in environmental water that had previously tested negative for $V$. cholerae $\mathrm{O} 1$ or $\mathrm{O} 139$ by the AST procedure. An aliquot of this dilution, as well as a dilution of stools in PBS, was cultured immediately to calculate the initial number of $V$. cholerae organisms present in the stools and to isolate the strain for further analysis. Because stools from cholera patients are known to contain lytic vibriophages, the presence of phage was also tested as described (14). Results shown in this study are from stools that were found negative for any lytic phage. The stool-spiked water was left for $6-16 \mathrm{~h}$ at room temperature before conducting the AST assays. Aliquots

1. Colwell, R. R. \& Spira, W. M. (1992) in Cholera, eds. Barua, D. \& Greenough, W. B., III (Plenum, New York), pp. 107-127.

2. Colwell, R. R. \& Huq, A. (1994) in Vibrio cholerae and Cholera: Molecular to Global Perspectives, eds. Wachsmuth, I. K., Blake, P. A. \& Olsvik, O. (Am. Soc. Microbiol., Washington, DC), pp. 117-133.

3. Pascual, M., Rodo, X., Ellner, S. P., Colwell, R. R. \& Bouma, M. J. (2000) Science 289, 1766-1769.

4. Faruque, S. M., Albert, M. J. \& Mekalanos, J. J. (1998) Microbiol. Mol. Biol. Rev. 62, 1301-1314.

5. Kaper, J. B., Morris, J. G. \& Levine, M. M. (1995) Clin. Microbiol. Rev. 8, 48-86.

6. Khan, M. U., Shahidullah, M., Haque, M. S. \& Ahmed, W. U. (1984) Trop. Geogr. Med. 36, 335-340.

7. Cash, R. A., Music, S. I., Libonati, J. P., Snyder, M. J., Wenzel, R. P. \& Hornick, R. B. (1974) J. Infect. Dis. 129, 45-52.

8. Levine, M. M., Kaper, J. B., Herrington, D., Losonsky, G., Morris, J. G., Clements, M. L., Black, R. E., Tell, B. \& Hall, R. (1988) Infect. Immun. 56, 161-167.

9. Roszak, D. B. \& Colwell, R. R. (1987) Appl. Environ. Microbiol. 53, 2889-2893.

10. Faruque, S. M., Chowdhury, N., Kamruzzaman, M., Dziejman, M., Rahman, M. H., Sack, D. A., Nair, G. B. \& Mekalanos, J. J. (2004) Proc. Natl. Acad. Sci. USA, 101, 2123-2128.

11. O’Toole, G., Kaplan, H. B. \& Kolter, R. (2000) Annu. Rev. Microbiol. 54, 49-79.

12. Reguera, G. \& Kolter, R. (2005) J. Bacteriol. 187, 3551-3555.

13. Zhu, J. \& Mekalanos, J. J. (2003) Dev. Cell 5, 647-656.

14. Faruque, S. M., Islam, M. J., Ahmad, Q. S., Faruque, A. S. G., Sack, D. A., Nair, G. B. \& Mekalanos, J. J. (2005) Proc. Natl. Acad. Sci. USA 102, 6119-6124.

15. Waldor, M. K., Tschape, H. \& Mekalanos, J. J. (1996) J. Bacteriol. 178, 4157-4165. of the same water, not inoculated with stools, were used as negative controls. Human studies protocols were reviewed and approved by the Research Review Committee and Ethical Review Committee at the International Centre for Diarrhoeal Disease Research, Bangladesh.

Infectivity Assay in Infant Mice. Stools from cholera patients were fractionated according to the size of suspended particles by centrifugation initially at $2000 \times g$ to precipitate cells bound to debris; the supernatant contained mostly planktonic cells. The precipitate was further washed in PBS ( $\mathrm{pH}$ 7.2) to remove any loosely adhering cells and was then resuspended in fresh PBS. The relative infectivity of $V$. cholerae cells present in different fractions of cholera stools was determined by the competition assay, in accordance with methods described by Merrell et al. (23). Competition assays were conducted by mixing the lacZ-negative reference strain DSM-V984 (23), grown in LB medium to stationary phase and logarithmic phase, with stool bacteria in a ratio of 1:10 (vol/vol). A total of 50 $\mu \mathrm{l}$ of a 1,000 -fold dilution of this mixture, representing $10^{4}$ to $10^{5}$ colony-forming units (cfu), was administered to 3- to 5-day-old Swiss Albino mice. After 20-24 h, the animals were killed and the small intestines were removed, homogenized, and plated on medium containing $1 \%$ peptone, $1 \% \mathrm{NaCl}, 3 \%$ gelatin, and $1.5 \%$ agar, supplemented with X-Gal to allow for subsequent enumeration of $\beta$-galactosidase-positive and $\beta$-galactosidase-negative $V$. cholerae colonies. Ratios of output bacteria were corrected for deviations in input ratios from 1:1, and the corrected ratios were termed the "competitive index" of the strains in question.

Probes and PCR Assays. The presence of virulence genes was determined by using specific PCR assays for the tcp $A$, tcpI, and $a c f B$ genes of the toxin-coregulated pilus pathogenicity island, and for the $\operatorname{ctx} A$ and zot genes of the CTX prophage (10). The SXT probe and the rRNA gene probe used in ribotyping have been described $(10,15)$. Southern blots were prepared and hybridized with the radioactively labeled probes, in accordance with standard methods (30).

This work was supported by National Institutes of Health Research Grant GM068851, under subagreements between the Harvard Medical School and the International Centre for Diarrhoeal Disease Research, Bangladesh (ICDDR,B). The ICDDR,B is supported by countries and agencies that share its concern for the health problems of developing countries.

16. Hochhut, B., Lotfi, Y., Mazel, D., Faruque, S. M., Woodgate, R. \& Waldor, M. K. (2001) Antimicrob. Agents Chemother. 45, 2991-3000.

17. Walsh, C. (2003) Antibiotics: Actions, Origins, Resistance (Am. Soc. Microbiol., Washington, DC), pp. 89-156.

18. Baker, E. M., Singleton, F. L. \& Hood, M. A. (1983) Appl. Environ. Microbiol. 46, 930-940.

19. Luria, S. \& Delbruck, M. (1943) Genetics 28, 491-511.

20. Spira, W. M., Sack, R. B. \& Froehlich, J. L. (1981) Infect. Immun. 32, 739-747.

21. Hase, C. C. (2000) Trends Microbiol. 8, 490-491.

22. Dibrov, P. (2005) Biochemistry (Moscow) 70, 150-153.

23. Merrell, D. S., Butler, S. M., Qadri, F., Dolganov, N. A., Alam, A., Cohen, M. B., Calderwood, S. B., Schoolnik, G. K. \& Camilli, A. (2002) Nature 417, 642-645.

24. Colwell, R. R., Huq, A., Islam, M. S., Aziz, K. M., Yunus, M., Khan, N. H., Mahmud, A., Sack, R. B., Nair, G. B., Chakraborty, J., et al. (2003) Proc. Natl. Acad. Sci. USA 100, 1051-1055.

25. Watnick, P. I., Fullner, K. J. \& Kolter, R. (1999) J. Bacteriol. 181, 3606-3609.

26. Meibom, K. L., Li, X. B., Nielsen, A. T., Wu, C. Y., Roseman, S. \& Schoolnik, G. K. (2004) Proc. Natl. Acad. Sci. USA 101, 2524-2529.

27. Faruque, S. M., Naser, I. B., Islam, M. J., Faruque, A. S. G., Ghosh, A. N., Nair, G. B., Sack, D. A. \& Mekalanos, J. J. (2005) Proc. Natl. Acad. Sci. USA. 102, 1702-1707.

28. Monsur, K. A. (1961) Trans. R. Soc. Trop. Med. Hyg. 55, 440-442.

29. World Health Organization (1974) World Health Organization Guidelines for the Laboratory Diagnosis of Cholera (Bacterial Disease Unit, W.H.O., Geneva).

30. Maniatis, T., Fritsch, E. F. \& Sambrook, J. (1982) Molecular Cloning: $A$ Laboratory Manual (Cold Spring Harbor Lab. Press, Woodbury, NY). 